\title{
Event-Triggered Control for a Three DoF Manipulator Robot
}

\section{(Control disparado por eventos para un robot manipulador de tres GDL)}

\author{
Saúl Enrique Benítez-García ${ }^{1}$, Miguel Gabriel Villarreal-Cervantes²
}

\begin{abstract}
:
In the classical approach of Time-Triggered Control (TTC), the control signal is updated at each sampling time as well as the system states to be controlled, which could imply a redundancy in the computational calculation as well as in the transfer of information in the regulation objective. On the other hand, the Event-Triggered Control (ETC) approach performs the same task in an asynchronous way, i.e,, it only updates the control signal when a performance requirement is violated and the states are updated at each sampling time. This reduces the amount of computational calculation without affecting the performance of the closed loop system. For this reason, in the present work the ETC is developed for the stabilization of a manipulator robot with three Degree of Freedom (DoF) in the joint space where a Lyapunov Control Function (LCF) is proposed to formulate the event function $\left(\mathrm{e}^{-}\right)$, which indicates whether or not is required the control signal updating. Simulation results show the reduction of the updates compared with a TTC.
\end{abstract}

Keywords: Event-Triggered Control; Time-Triggered Control; Manipulator Robot; Lyapunov Control Function; Event Function.

\begin{abstract}
Resumen:
En el enfoque clásico de control disparado por tiempo (del inglés TTC), en cada instante de muestreo se actualiza de manera síncrona la señal de control así como los estados del sistema a controlar, lo que podría implicar en una redundancia en el cálculo computacional así como en la transferencia de información en el objetivo de regulación. Por otro lado, el enfoque de control disparado por eventos (del inglés ETC ) realiza la misma tarea de manera asíncrona, es decir, solo actualiza la señal de control cuando se viola algún requisito de rendimiento y los estados son actualizados en cada instante de muestreo. Esto reduce la cantidad de cálculo computacional sin afectar el rendimiento del sistema en lazo cerrado. Por tal motivo, en el presente trabajo se desarrolla el ETC para la estabilización de un robot manipulador en el espacio articular, donde la función de evento $(\bar{e})$, que indica si se requiere o no actualizar la señal de control, se basa en una Función de Control de Lyapunov (FCL), lo que asegura convergencia asintótica del error a cero. EI ETC se verifica en experimentos en simulación, comparando los resultados con una estrategia de control realizada bajo el enfoque TTC.
\end{abstract}

Palabras clave: Control disparado por eventos; Robot manipulador; Función de Control de Lyapunov; Función de evento.

\footnotetext{
${ }^{1}$ Instituto Politécnico Nacional, CIDETEC, Departamento de Posgrado, Ciudad de México - México (sbenitezg1100@alumno.ipn.mx).

2 Instituto Politécnico Nacional, CIDETEC, Departamento de Posgrado, Ciudad de México - México (mvillarrealc@ipn.mx).
} 


\section{Introduction}

In recent years, technological advances in computer systems and sensors has lead in the development and application of advanced control theories and robotics. These advances are presented jointly, since the nonlinear models of robots have served as a good study case in order to illustrate the general concepts of analysis and design of advanced control theories (Canudas de Wit, Siciliano, \& Bastin, 1996), for example: adaptive control (Tso \& Lin, 1996), sliding modes control (Zhao, Sheng, \& Liu, 2014), Lyapunov based control (Halalchi, Bara, \& Laroche, 2010), nonlinear predictive control (Wilson, Charest, \& Dubay, 2016), fuzzy logic control (Chen, Wang, Zhai, \& Gao, 2017), among others. The main reason lies in its ability to manipulate materials, parts, tools or specialized devices by programming their movements.

It is well known that implementation of control theories in digital systems is possible by two kinds of control models: TTC and ETC. The first model consists of the measurement of system parameters uniformly in time with a sampling period $T$, and likewise has to update the signal control periodically for every time instant $t_{k}=k T \forall k=1,2,3 \ldots$ (Durand, and Guerrero-Castellanos, Marchand, \& Guerrero-Sánchez, 2013). Furthermore, this model can be separated in two ways: continuous control by emulation and digital control. The continuous control by emulation is possible, if and only if, an enough small sampling time is guaranteed to ensure acceptable system performance. However, this constraint cannot always be guaranteed for all systems, due to the sampling devices and computer systems may present delays and errors of digitalization. On the other hand, the digital control is a mature and well known field for linear systems. However, when this is applied to nonlinear systems it may cause instability in the system because the digital control is based on transforming the continuous time system to discrete time, and afterwards to design a control law in discrete time. This process requires obtaining analytical nonlinear models in exact discrete time which implies solving a nonlinear explicit initial value problem (Monaco \& Normand-Cyrot, 2007). The second approach is based on the execution of the control strategy by activating the event function. The activation of the event function occurs when a system performance constraint is violated.

The ETC offers stability and a decrease in the number of control signal updates. As a result, the computational load decreases as at the same time as the energy consumption. Consequently, the ETC have been applied in some works: in (Villarreal-Cervantes, Guerrero-Castellanos, Ramírez-Martínez, \& Sánchez-Santana, 2015) a comparison between an ETC and a Calculated Torque Control (CTC) are presented for the (3.0) mobile robot. The experimental results indicate a decrease of $23.73 \%$ in the number of updates of the ETC signal is obtained, compared to that required by the CTC. In (Tripathy, Kar, \& Paul, 2014 ) the design of an ETC strategy based on robust control is proposed. This is validated by simulation in a SCARA type robot with two degrees of freedom, where the results showed asymptotic convergence with or without the presence of some disturbance. In (Durand, and Guerrero-Castellanos, Marchand, \& Guerrero-Sánchez, 2013) the stabilization of an inverted pendulum by means of an ETC strategy is presented where the activation mechanism, based on the Lyapunov stability approach, is obtained through the methodology in (Marchand, Durand, \& Guerrero Castellanos, 2013). Experiments and analysis of results in real time showed an approximate reduction between $98 \%$ and $50 \%$; this compared to the classic scheme presented in the TTC.

Despite the benefits provided by the ETC, few results have been reported in the framework of robotics and mechatronics such as those mentioned above. For this reason, in the present work an ETC for the regulation of a robot manipulator with three DoF, which includes gravitational terms, is proposed. The strategy of ETC is based on the dynamic model of the manipulator robot; for this reason, in Section 2 the model is presented in state space. Likewise, the mathematical preliminaries concerning the stabilization of nonlinear systems under the Event-Triggered approach are given. In Section 3, the existence of a Lyapunov Control Function is shown as well as its mathematical proof. In addition, the event 
function that triggers the ETC strategy based on a CTC for the manipulator robot is developed. Comparative results of the ETC with a CTC is performed in Section 4. Finally, the conclusions of the present work are drawn in Section 5.

\section{Mathematical Preliminaries}

In the next Section, the dynamic model of the manipulator robot with three degrees of freedom is shown. Likewise, some relevant aspects on stabilization of nonlinear systems through the ETC are illustrated. These preliminaries will be necessary for the further development of the ETC, which will be used to stabilize the system at some desired point.

According to Kelly and Loria (Kelly, Santibáñez, \& Loría, 2005), a manipulator robot is an articulated mechanical arm composed of links interconnected through joints, which allow a relative movement between two consecutive links.

\section{Manipulator robot dynamic model}

Figure 1 shows the schematic diagram of the manipulator robot, which consists of three revolute joints. The dynamic and kinematic parameters of the $i$-th link are given by the distance between the axis of rotation to the center of mass $l_{c_{i}}$, the inertia $I_{z_{i}}$, the mass $m_{i}$, and the link length $l_{i}$, with $i=1,2,3$.

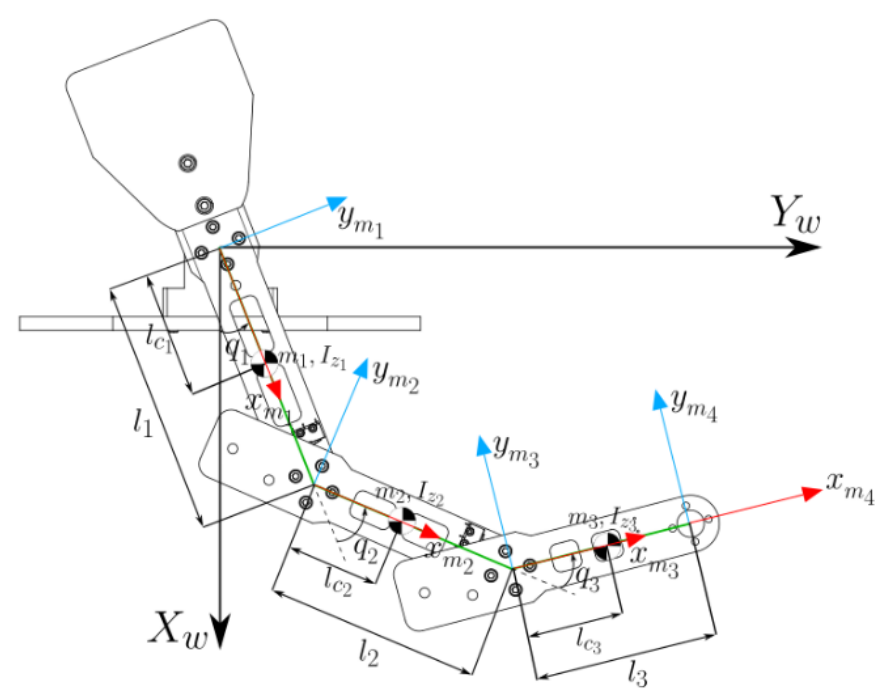

Figure 1. Schematic diagram of the manipulator robot.

Let Equation 1 the representation in state variables of the dynamic model of the manipulator robot, where $x=\left[x_{1}, x_{2}, x_{3}, x_{4}, x_{5}, x_{6}\right]^{T}=[q, \dot{q}]^{T}=\left[q_{1}, q_{2}, q_{3}, \dot{q}_{1}, \dot{q}_{2}, \dot{q}_{3}\right]^{T} \in$ $\mathbb{R}^{6}$ is the state vector corresponding to the angular position $q \in \mathbb{R}^{3}$ and velocity $\dot{q} \in \mathbb{R}^{3}$ vectors, expressed in the joint space for each degree of freedom.

$$
\dot{x}=f(x)+g(x) u
$$

where:

$$
\begin{aligned}
& u=\left[u_{1}, u_{2}, u_{3}\right]^{T} \in \mathbb{R}^{3} . \\
& f(x)=\left[\begin{array}{c}
{\left[x_{4}, x_{5}, x_{6}\right]^{T}} \\
M^{-1}\left[-C\left[x_{4}, x_{5}, x_{6}\right]^{T}-G\right.
\end{array}\right] \in \mathbb{R}^{6} . \\
& g(x)=\left[\begin{array}{c}
0 \in \mathbb{R}^{3 \times 3} \\
M^{-1}
\end{array}\right] \in \mathbb{R}^{6}
\end{aligned}
$$


The elements of the inertial matrix $M \in \mathbb{R}^{3 \times 3}$ are:

$$
\begin{aligned}
& M_{11}=I_{z_{1}}+I_{z_{2}}+I_{z_{3}}+l_{1}^{2} m_{2}+l_{1}^{2} m_{3}+l_{2}^{2} m_{3}+l_{c_{1}}^{2} m_{1}+\mathrm{l}_{\mathrm{c}_{2}}^{2} \mathrm{~m}_{2}+\mathrm{l}_{\mathrm{c}_{3}}^{2} \mathrm{~m}_{3}+2 \mathrm{l}_{1} \mathrm{l}_{2} \mathrm{~m}_{3} \bar{\beta}_{1} \\
& +2 \mathrm{l}_{1} \mathrm{l}_{\mathrm{c}_{2}} \mathrm{~m}_{2} \bar{\beta}_{1}+2 \mathrm{l}_{1} \mathrm{l}_{\mathrm{c}_{2}} \mathrm{~m}_{2} \bar{\beta}_{1}+2 \mathrm{l}_{2} \mathrm{l}_{\mathrm{c}_{3}} \mathrm{~m}_{3} \bar{\beta}_{2}+2 \mathrm{l}_{1} \mathrm{l}_{\mathrm{c}_{3}} \mathrm{~m}_{3} \bar{\beta}_{3} \\
& M_{12}=I_{z_{2}}+I_{z_{3}}+l_{2}^{2} m_{3}+2 l_{2} l_{c_{3}} m_{3} \bar{\beta}_{2}+l_{1} l_{2} m_{3} \bar{\beta}_{1}+\mathrm{l}_{\mathrm{c}_{2}}^{2} \mathrm{~m}_{2}+\mathrm{l}_{\mathrm{c}_{3}}^{2} \mathrm{~m}_{3}+\mathrm{l}_{1} \mathrm{l}_{\mathrm{c}_{2}} \mathrm{~m}_{2} \bar{\beta}_{1} \\
& +\mathrm{l}_{1} \mathrm{l}_{\mathrm{c}_{3}} \mathrm{~m}_{3} \bar{\beta}_{3} \\
& M_{13}=I_{z_{3}}+l_{c_{3}}^{2} m_{3}+l_{1} l_{c_{3}} m_{3} \bar{\beta}_{3}+l_{2} l_{c_{3}} m_{3} \bar{\beta}_{2} \\
& M_{21}=I_{z_{2}}+I_{z_{3}}+l_{2}^{2} m_{3}+2 l_{2} l_{c_{3}} m_{3} \bar{\beta}_{2}+l_{1} l_{2} m_{3} \bar{\beta}_{1}+l_{c_{2}}^{2} m_{2}+l_{c_{3}}^{2} m_{3}+l_{1} l_{c_{2}} m_{2} \bar{\beta}_{1} \\
& +l_{1} l_{c_{3}} m_{3} \bar{\beta}_{3} \\
& M_{22}=I_{z_{2}}+I_{z_{3}}+l_{2}^{2} m_{3}+2 l_{2} l_{c_{3}} m_{3} \bar{\beta}_{2}+l_{c_{2}}^{2} m_{2}+l_{c_{3}}^{2} m_{3} \\
& M_{23}=I_{z_{3}}+l_{c_{3}}^{2} m_{3}+l_{2} l_{c_{3}} m_{3} \bar{\beta}_{2} \\
& M_{31}=I_{z_{3}}+l_{c_{3}}^{2} m_{3}+l_{1} l_{c_{3}} m_{3} \bar{\beta}_{3}+l_{2} l_{c_{3}} m_{3} \bar{\beta}_{2} \\
& M_{32}=I_{z_{3}}+l_{c_{3}}^{2} m_{3}+l_{2} l_{c_{3}} m_{3} \bar{\beta}_{2} \\
& \text { M_33 }=I_{z_{3}}+l_{c_{3}}^{2} m_{3}
\end{aligned}
$$

The elements of the Coriolis and Centrifugal matrix $C \in \mathbb{R}^{3 \times 3}$ are represented by:

$$
\begin{aligned}
& C_{11}=-x_{5} l_{1} l_{2} m_{3} \bar{\alpha}_{2}-x_{5} l_{1} l_{c_{2}} m_{2} \bar{\alpha}_{2}-x_{6} l_{2} l_{c_{3}} m_{3} \bar{\alpha}_{3}-x_{5} l_{1} l_{c_{3}} m_{3} \bar{\alpha}_{5}-x_{6} l_{1} l_{c_{3}} m_{3} \bar{\alpha}_{5} \\
& C_{12}=-x_{4} l_{1} l_{2} m_{3} \bar{\alpha}_{2}-x_{4} l_{1} l_{c_{2}} m_{2} \bar{\alpha}_{2}-x_{5} l_{1} l_{2} m_{3} \bar{\alpha}_{2}-x_{6} l_{2} l_{c_{3}} m_{3} \bar{\alpha}_{3}-x_{4} l_{1} l_{c_{3}} m_{3} \bar{\alpha}_{5} \\
& -x_{5} l_{1} l_{c_{3}} m_{3} \bar{\alpha}_{5}-x_{6} l_{1} l_{c_{3}} m_{3} \bar{\alpha}_{5}-x_{5} l_{1} l_{-} c_{2} m_{2} \bar{\alpha}_{2} \\
& C_{13}=-x_{4} l_{2} l_{c_{3}} m_{3} \bar{\alpha}_{3}-x_{5} l_{2} l_{c_{3}} m_{3} \bar{\alpha}_{3}-x_{6} l_{2} l_{c_{3}} m_{3} \bar{\alpha}_{3}-x_{4} l_{1} l_{c_{3}} m_{3} \bar{\alpha}_{5}-x_{5} l_{1} l_{c_{3}} m_{3} \bar{\alpha}_{5} \\
& -x_{6} l_{1} l_{c_{3}} m_{3} \bar{\alpha}_{5} \\
& C_{21}=x_{4} l_{1} l_{c_{3}} m_{3} \bar{\alpha}_{5}+x_{4} l_{1} l_{2} m_{3} \bar{\alpha}_{-} 2+x_{4} l_{1} l_{c_{2}} m_{2} \bar{\alpha}_{2}-x_{6} l_{2} l_{c_{3}} m_{3} \bar{\alpha}_{3} \\
& C_{22}=-x_{6} l_{2} l_{c_{3}} m_{3} \bar{\alpha}_{3} \\
& C_{23}=-x_{4} l_{2} l_{c_{3}} m_{3} \bar{\alpha}_{3}-x_{5} l_{2} l_{c_{3}} m_{3} \bar{\alpha}_{3}-x_{6} l_{2} l_{c_{3}} m_{3} \bar{\alpha}_{3} \\
& C_{31}=x_{4} l_{2} l_{c_{3}} m_{3} \bar{\alpha}_{3}+x_{4} l_{1} l_{c_{3}} m_{3} \bar{\alpha}_{5}+x_{5} l_{2} l_{c_{3}} m_{3} \bar{\alpha}_{3} \\
& C_{32}=x_{4} l_{2} l_{c_{3}} m_{3} \bar{\alpha}_{3}+x_{5} l_{2} l_{c_{3}} m_{3} \bar{\alpha}_{3} \\
& C_{33}=0
\end{aligned}
$$

The elements of the gravity vector $G \in \mathbb{R}^{3}$ are given as:

$$
\begin{array}{rlrl}
G_{11} & =g l_{2} m_{3} \bar{\alpha}_{4}+g l_{c_{2}} m_{2} \bar{\alpha}_{4}+g l_{1} m_{2} \bar{\alpha}_{1}+g l_{1} m_{3} \bar{\alpha}_{1}+g l_{c_{1}} m_{1} \bar{\alpha}_{1}+g l_{c_{3}} m_{3} \bar{\alpha}_{6} \\
G_{21} & =g l_{2} m_{3} \bar{\alpha}_{4}+g l_{c_{2}} m_{2} \bar{\alpha}_{4}+g l_{c_{3}} m_{3} \bar{\alpha}_{6} & \\
G_{31}=g l_{c_{3}} m_{3} \bar{\alpha}_{6} & \bar{\alpha}_{1}=\sin \left(x_{1}\right) & \bar{\alpha}_{-} 4=\sin \left(x_{1}+x_{2}\right) \\
\bar{\beta}_{1}=\cos \left(x_{2}\right) & \bar{\alpha}_{2}=\sin \left(x_{2}\right) & \bar{\alpha}_{5}=\sin \left(x_{2}+x_{3}\right) \\
\bar{\beta}_{2}=\cos \left(x_{3}\right) & \bar{\alpha}_{3}=\sin \left(x_{3}\right) & \bar{\alpha}_{6}=\sin \left(x_{1}+x_{2}+x_{3}\right) \\
\bar{\beta}_{3}=\cos \left(x_{2}+x_{3}\right) & \left.x_{3}\right)
\end{array}
$$

\section{General formula of Event-Triggered Control}

The ETC approach is restricted to the study of dynamic systems that have the form shown in Equation 2, where $x \in X \subset \mathbb{R}^{n}, u \in \mathcal{U} \subset \mathbb{R}^{p}, f(x)$ and $g(x)$ are smooth Lipschitz functions that vanish at the origin.

$$
\dot{x}=f(x)+g(x) u
$$

In the present work the stabilization case at the origin has been considered. If the system supports a state feedback $k: \mathcal{X} \rightarrow \mathcal{U}$ which stabilizes the system asymptotically, then there exists a LCF $V: \mathcal{X} \rightarrow \mathbb{R}$, which is a smooth and positive defined function, resulting in Equation 3. 


$$
\dot{V}=\frac{\partial V}{\partial x} f(x)+\frac{\partial V}{\partial x} g(x) k(x)
$$

The ETC approach in general requires two functions

- Event function $\bar{e}: \mathcal{X} \times \mathcal{X} \rightarrow \mathbb{R}$ indicating whether it is necessary to update $(\bar{e} \leq$ $0)$ or not $(\bar{e}>0)$ the control signal. The event function $\bar{e}$ uses the current state vector $x$ as input, and a memory parameter $m$ from the vector $x$ corresponding to the last instant of time in which an event function $\bar{e}$ became negative.

- Feedback function: A state feedback is when $k: \mathcal{X} \rightarrow \mathcal{U}$. This function is calculated, if and only if, the event function is activated.

Definition1. (Marchand, Durand, \& Guerrero Castellanos, 2013): An ETC $(\bar{e}, k)$ is said to be semi-uniformly MSI (Minimal Sampling Interval property) if for all $\delta>0$ and all $x_{0}$ on the radio sphere $\delta$ with center at the origin $\mathcal{B}(\delta)$, the time interval between two consecutive events can be bounded below by some $\underline{\tau}>0$.

It is well known that for nonlinear systems of the form (2) with a ETC $(\bar{e}, k)$ semiuniform MSI, the solution for Equation 2, with initial conditions $x_{0} \in \mathcal{X}$ at the instant $t=0$, is defined for all $t$ positive as the solution to the differential system in Equations 4 and 5 .

$$
\begin{gathered}
\dot{x}=f(x)+g(x) k(m) \\
m= \begin{cases}x & \text { if } \bar{e}(x, m) \leq 0, x \neq 0 \\
0 & \text { elsewhere }\end{cases} \\
\text { with } x(0)=x_{0} \text { and } m(0)=x(0)
\end{gathered}
$$

Theorem 1. (Universal Event-Triggered formula (Marchand, Durand, \& Guerrero Castellanos, 2013)): If there exists a LFC for the system (2), then the event-based feedback $(\bar{e}, k)$ defined above is semi-uniform MSI smooth in $\mathcal{X}$ such that we have Equation 6.

$$
\frac{\partial V}{\partial x} f(x)+\frac{\partial V}{\partial x} g(x) k(x)<0, \quad x \in X
$$

where $m$ is defined in Equation 5 and, the feedback control $k$ and the event function $\bar{e}$ is given by Equations 7 and 8 respectively.

$$
\begin{gathered}
k(m)=-b(x) \delta(x) \gamma(x) \in \mathbb{R}^{n} \\
\bar{e}(x, m)=-a(x)-b(x) k(m)-\sigma \sqrt{a(x)^{2}+\bar{\theta}(x) b(x) \Delta(x) b(x)^{T}} \in \mathbb{R}
\end{gathered}
$$

with:

- $\quad a(x)=\frac{\partial V}{\partial x} f(x)$ and $=\frac{\partial V}{\partial x} g(x)$

- $\Delta(x): x \rightarrow \mathbb{R}^{p \times p}$ wich that $\Delta(x)=\operatorname{diag}\left(\delta_{1}(x), \delta_{2}(x), \ldots, \delta_{p}(x)\right)$ is a smooth and definite positive function in $\mathcal{S}=\{x \in \mathcal{X}\|\| b(x) \| \neq 0$

- $\bar{\theta}(x): X \rightarrow \mathbb{R}$ is a smooth function, such that $\bar{\theta}(x)\|\Delta(x)\|$ vanishes at the origin and ensures the inequality $a(x)^{2}+\bar{\theta}(x) b(x) \Delta(x) b(x)^{T}>0$ in $\delta$.

- $\sigma$ is an adjustable control parameter in $[0,1)$.

- $\gamma(x) \rightarrow \mathbb{R}$ is defined by Equation 9 . 


$$
\gamma(x)=\left\{\begin{array}{c}
\frac{\sigma \sqrt{a(x)^{2}+\bar{\theta}(x) b(x) \Delta(x) b(x)^{T}}}{b(x) \Delta(x) b(x)^{T}} \text { if } x \in \mathcal{S} \\
0 \text { if } x \notin \mathcal{S}
\end{array}\right.
$$

\section{Design Control Strategy}

In this section the design of the ETC strategy for the stabilization of the manipulator robot is described.

\section{Lyapunov Control Function}

Considering the regulation problem and a variable change $[e, \dot{q}]$, the Lyapunov Function $V: \mathbb{R}^{6} \rightarrow \mathbb{R}$ defined in Equation 10 is proposed, where $e=\left[q-q_{d}\right]^{T}$ is the error between the desired angular position $q_{d}=\left[q_{d_{1}}, q_{d_{2}}, q_{d_{3}}\right]$ and the real one and $P_{1}, P_{2}$ and $P_{3} \in \mathbb{R}^{3 \times 3}$ are symmetric and positive defined matrices.

$$
V(e, \dot{q})=\frac{1}{2} e^{T} P_{1} e+\frac{1}{2} \dot{q}^{T} P_{2} \dot{q}+e^{T} P_{3} \dot{q}
$$

Considering the control system in Equation 11, then $V$ is a LCF for the system shown in Equation 1 relative to the equilibrium point $[e, \dot{q}]^{T}=[0,0]^{T}$.

$$
u=M(q) \bar{v}+C(q, \dot{q}) \dot{q}+G(q)
$$

with:

$$
\bar{v}=-\frac{1}{4} R^{-1} B^{T}\left[\begin{array}{ll}
P_{1} & P_{3} \\
P_{3} & P_{2}
\end{array}\right]\left[\begin{array}{l}
e^{q} \\
\dot{q}
\end{array}\right]
$$

where $R=\operatorname{diag}\left[r_{1}, r_{2}, r_{3}\right] \in \mathbb{R}^{3 \times 3}$ is a positive definite gains matrix and $B$ is given by $B=$ $\left[0 \in \mathbb{R}^{3 \times 3}, I \in \mathbb{R}^{3 \times 3}\right]^{T}$.

As a result of applying the control strategy shown in Equation 11 to the dynamic system in Equation 1, the system is asymptotically stable, so that $V(e, \dot{q})$ is a LCF for that system. It is worth mentioning that, in the present work requires the control strategy shown in Equation 11 in order to obtain a closed-loop linear system and hence the feedback control in Equation 7 is not used anymore and only the event function of the ETC depends on the LCF.

The proof is given in Appendix $A$.

\section{Event function for the manipulator robot}

Once the LCF is established, it is possible to develop the ETC methodology proposed in (Marchand, Durand, \& Guerrero Castellanos, 2013). In order to obtain the functions $a(x)$ and $b(x)$, necessary for the event function in Equation 8; the temporal derivative of the LCF in Equation 10 is taken again evaluated along the trajectory in Equation 1, as seen in Equation 12.

$$
\dot{V}(x)=\left[\begin{array}{ll}
e & \dot{q}
\end{array}\right]\left[\begin{array}{ll}
P_{1} & P_{3} \\
P_{3} & P_{2}
\end{array}\right] f(x)+\left[\begin{array}{ll}
e & \dot{q}
\end{array}\right]\left[\begin{array}{ll}
P_{1} & P_{3} \\
P_{3} & P_{2}
\end{array}\right] g(x) u(m)
$$


Therefore, the functions $\mathrm{a}(\mathrm{x})$ and $\mathrm{b}(\mathrm{x})$ are given by Equations 13 and 14 .

\section{Results}

$$
\begin{aligned}
& a(x)=\left[\begin{array}{ll}
e & \dot{q}
\end{array}\right]\left[\begin{array}{ll}
P_{1} & P_{3} \\
P_{3} & P_{2}
\end{array}\right]\left[\begin{array}{c}
\dot{q} \\
M^{-1}[-C \dot{q}-G
\end{array}\right] \\
& b(x)=\left[\begin{array}{ll}
e & \dot{q}
\end{array}\right]\left[\begin{array}{ll}
P_{1} & P_{3} \\
P_{3} & P_{2}
\end{array}\right]\left[\begin{array}{c}
0 \in \mathbb{R}^{3 \times 3} \\
M^{-1}
\end{array}\right] u(m)
\end{aligned}
$$

In the current section, the operation of the ETC strategy applied to a three DoF manipulator robot is analyzed, considering the regulation problem in the joint space. For this purpose, a comparison is made between the effectiveness of the ETC and a Computed Torque Control for the stabilization of the manipulator robot. The manipulator robot parameters are shown in Table 1.

Table 1. Three DoF manipulator robot specifications.

\begin{tabular}{|c|c|c|c|}
\hline Parameter & Description & Value & Units \\
\hline$l_{1}$ & Link length 1 & 0.18 & $\mathrm{~m}$ \\
\hline$l_{2}$ & Link length 2 & 0.15 & $\mathrm{~m}$ \\
\hline$l_{3}$ & Link length 3 & 0.13 & $\mathrm{~m}$ \\
\hline$l_{C_{1}}$ & Link $\underline{\text { mass }}$ center length 1 & -0.04835 & $\mathrm{~m}$ \\
\hline$l_{C_{2}}$ & Link $\underline{\text { mass }}$ center length 2 & 0.09496 & $\mathrm{~m}$ \\
\hline$l_{C_{3}}$ & Link $\underline{\text { mass }}$ center length 3 & 0.01222 & $\mathrm{~m}$ \\
\hline$I_{z_{1}}$ & Link $\underline{\text { Inertial }}$ momentum 1 & 0.05187067811 & $\mathrm{kgm}^{2}$ \\
\hline$I_{z_{2}}$ & Link $\underline{\text { Inertial }} \underline{\text { momentum 2 }}$ & 0.00559463172 & $\mathrm{kgm}^{2}$ \\
\hline$I_{z_{3}}$ & Link $\underline{\text { Inertial }} \underline{\text { momentum 3 }}$ & 0.00106098215 & $\mathrm{kgm}$ \\
\hline$m_{1}$ & Link $\underline{\text { mass 1 }}$ & 5.35884 & $\mathrm{~kg}$ \\
\hline$m_{2}$ & Link $\underline{\text { mass 2 }}$ & 0.97433 & $\mathrm{~kg}$ \\
\hline$m_{3}$ & Link $\underline{\text { mass 3 }}$ & 0.38986 & $\mathrm{~kg}$ \\
\hline
\end{tabular}

To carry out experiments in simulation, the rest position is considered as the initial condition for the manipulator robot, i.e., $x(0)=[0,0,0,0,0,0]^{T}$. In addition, four different desired angular positions have been taken into account: $Q_{1}=\left[0,-\frac{\pi}{3},-\frac{\pi}{4}, 0,0,0\right]^{T}, Q_{2}=$ $\left[\frac{\pi}{4},-\frac{\pi}{4},-\frac{\pi}{3}, 0,0,0\right]^{T}, Q_{3}=\left[\frac{\pi}{2}, 0,0,0,0,0\right]^{T}$ and $Q_{-} 4=\left[\frac{5 \pi}{6},-\frac{\pi}{3}, \frac{\pi}{2}, 0,0,0\right]^{T}$, such that the manipulator robot reaches them in sequential order. These positions must be reached in a maximum time of $15 \mathrm{~s}$, therefore, the final simulation time will be $t_{f}=60 \mathrm{~s}$. The experiments were performed in Matlab, with a fixed sampling time of $\Delta t=5 \mathrm{~ms}$.

On the other hand, the proposed parameters for the ETC are the following: i) event frequency $\sigma=0.8$ and ii) functions $\bar{\theta}(x)=b(x) \Delta(\mathrm{x}) b(x)^{T}-2 a(x)$ and $\Delta(x)=$ $\operatorname{diag}[1,1,1]$. As noted above, the gains $R, P_{1}, P_{2}$ and $P_{-} 3$ are parameters in common between the two control strategies ETC and CTC. Therefore, these parameters are shown below: i) matrix of gains $R=\operatorname{diag}(0.3,0.15,0.1)$, obtained based on tests in simulation and ii) matrix of gains $P_{1}=P_{2}=\operatorname{diag}(1.73205,1.73205,1.73205)$ and $P_{3}=\operatorname{diag}(1,1,1)$, obtained from the "care" function of Matlab, which calculates the solution of the Riccati Algebraic equation in continuous time. Those gains are used in both control strategies in order to make a fair comparison.

Figure 2a represents the behavior of the end effector of the manipulator robot in the workspace $X_{w}-Y_{w}$ and in Figures $2 b-2 d$ displays the angular position of each link for both control strategies. Similar behavior in both control strategies is observed. In order to provide a quantitative results three performance indices are considered in Table 2. Those indices 
are Integral Absolute Error (AIE), the Integral Time-weighted Absolute Error (ITAE) and the Integral Square Error (ISE). It is clear that the CTC presents a better performance than the ETC in the specified task. In addition, it is possible to deduce that the error converges to zero in both control approaches. These results indicate that the ETC system does not significantly impair closed loop performance.

Table 2. ETC and CTC comparative results.

\begin{tabular}{|c|c|c|c|c|c|c|}
\hline \multirow[t]{2}{*}{$\begin{array}{l}\text { Angular } \\
\text { Position }\end{array}$} & \multicolumn{2}{|c|}{$\begin{array}{c}\text { IAE } \\
\text { Control law }\end{array}$} & \multicolumn{2}{|c|}{$\begin{array}{c}\text { ITAE } \\
\text { Control law } \\
\end{array}$} & \multicolumn{2}{|c|}{$\begin{array}{c}\text { ISE } \\
\text { Control law }\end{array}$} \\
\hline & ETC & CTC & ETC & CTC & ETC & CTC \\
\hline$x_{1}$ & 4.3129 & 4.5208 & 137.2080 & 148.0418 & 2.2021 & 2.4086 \\
\hline$x_{2}$ & 11.3053 & 10.1552 & 197.6659 & 182.2721 & 10.5123 & 9.4270 \\
\hline$x_{3}$ & 8.4430 & 9.0382 & 233.9371 & 239.3566 & 6.5974 & 6.9510 \\
\hline Total & 24.0613 & 23.7142 & 568.8110 & 569.670 & 19.3119 & 18.7866 \\
\hline $\begin{array}{c}\text { Energy } \\
\text { Consumntion }\end{array}$ & \multicolumn{6}{|c|}{$\left(\left|u_{1}(t)\right|+\left|u_{2}(t)\right|+\left|u_{3}(t)\right|\right) d t$} \\
\hline & \multicolumn{3}{|c|}{ ETC } & \multicolumn{3}{|c|}{ CTC } \\
\hline$u$ & \multicolumn{3}{|c|}{128.1302} & \multicolumn{3}{|c|}{128.2731} \\
\hline
\end{tabular}

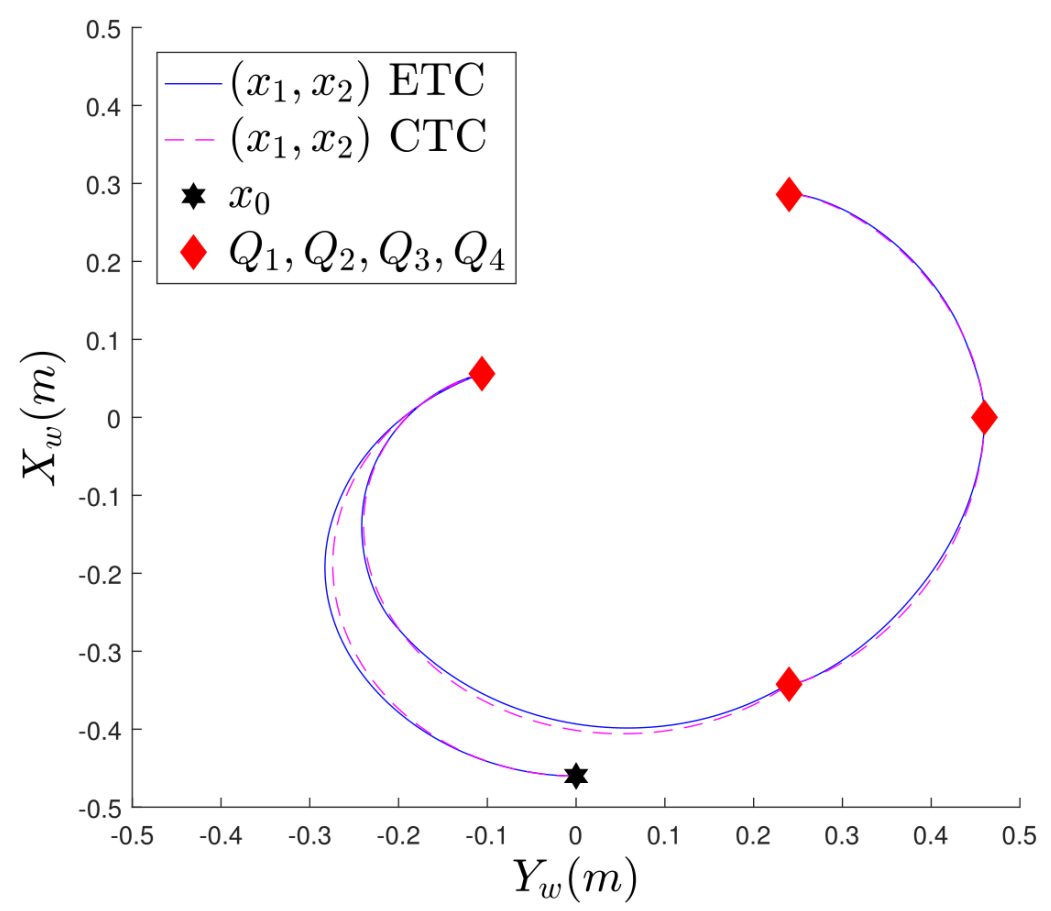

a) Behaviour in $X_{w}-Y_{w}$ plane

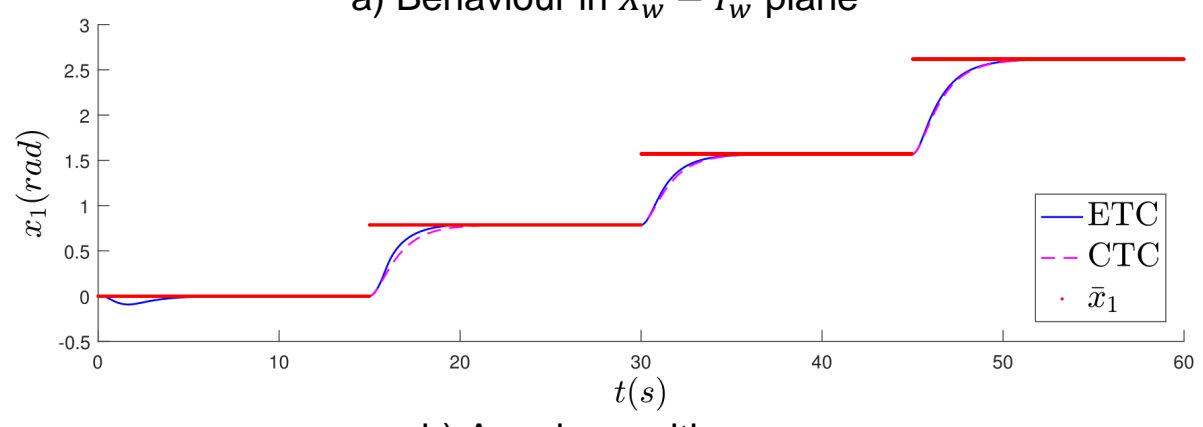

b) Angular position $x_{1}$ 


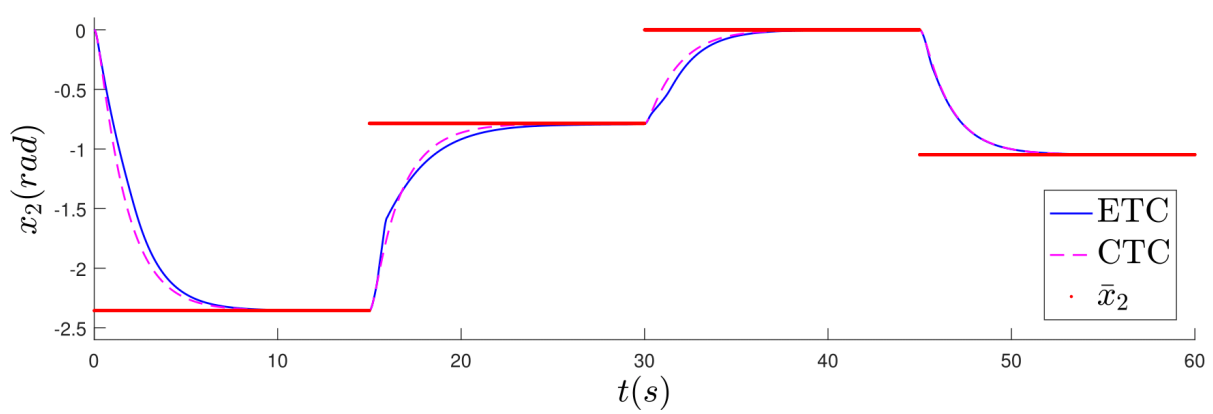

c) Angular position $x_{2}$

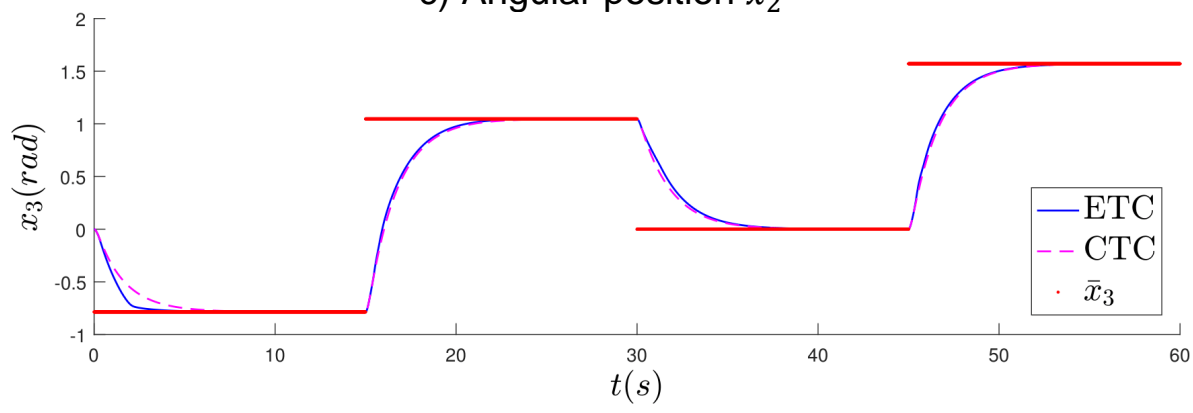

d) Angular position $x_{3}$

Figure 2. Behaviour of the three DoF manipulator robot with a ETC and a CTC in the joint space

In relation to the respective control signals for both control strategies, in Figure 3 their behavior are shown. To evaluate the energy consumption performance of both strategies, in the last row of Table 2 the total torque required to control the manipulator robot is given, which results that the ETC consumes a lower energy than the CTC.

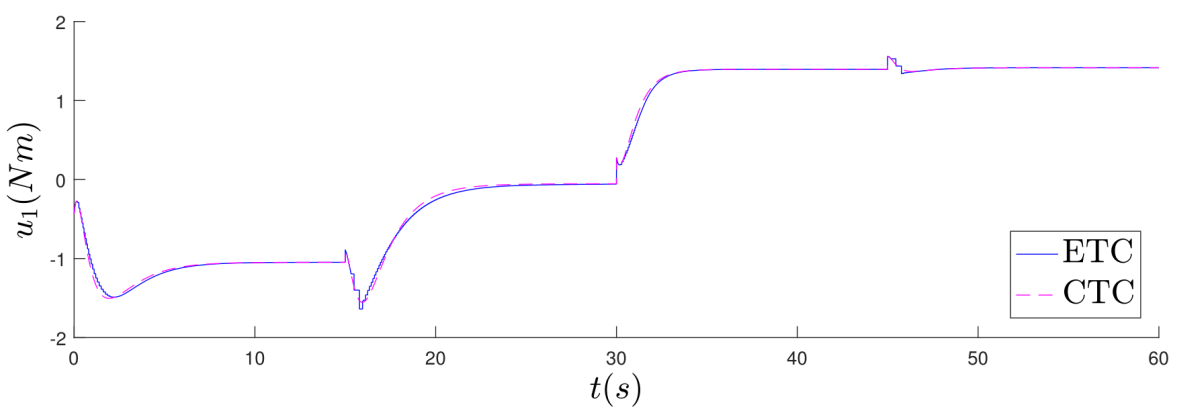

a) Control signal $u_{1}$

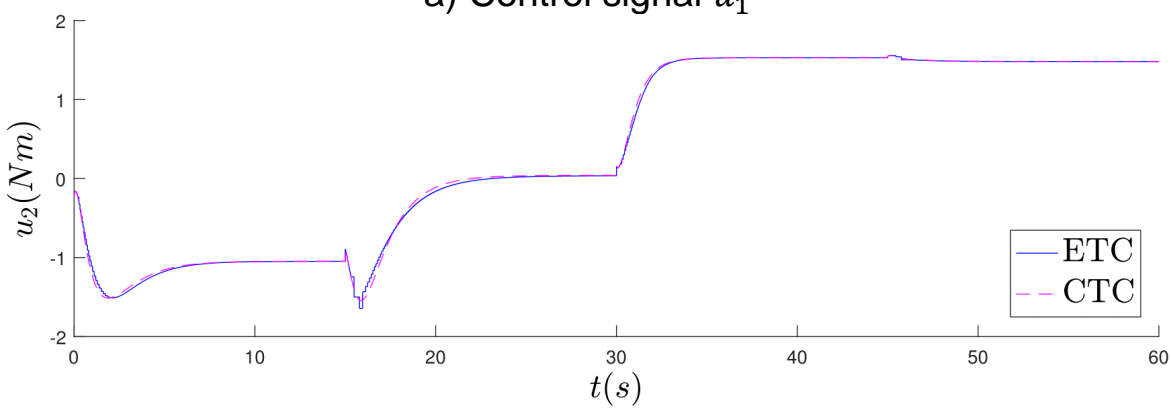

b) Control signal $u_{2}$ 


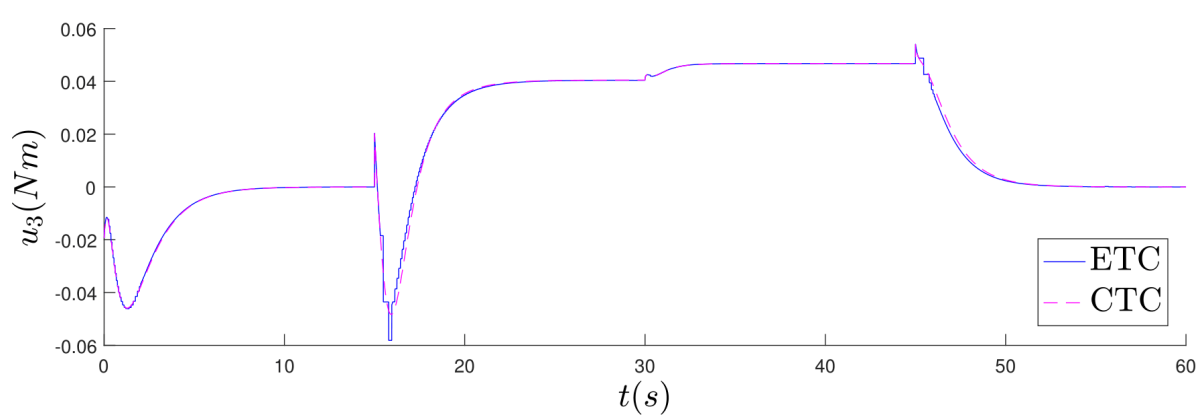

c) Control signal $u_{3}$

Figure 3. Control performance signals of the ETC and the CTC in the regulation problem

Finally, in the Figure 4a the Lyapunov function is shown, where the function grows when a change between positions is required, and the convergence around zero occurs when the system stabilizes at the desired position; similarly, this behavior is presented in the event function shown in Figure $4 b$. Furthermore, Figure $4 c$ shows the event flag, where "1" ( $\bar{e} \leq 0)$ indicates the update of the control signal and " 0 " ( $\bar{e}>0)$ means that the previous control signal is used.

Taking the sampling time of $\Delta t=5 \mathrm{~ms}$, the classical approach of CTC based on TTC requires a total of 12000 control signal updates, meanwhile the ETC updates 8060 times the control signal. Therefore, the ETC decreases by $32.8389 \%$ the number of required updates compared to the CTC. Consequently, computational calculation and energy consumption is also reduced

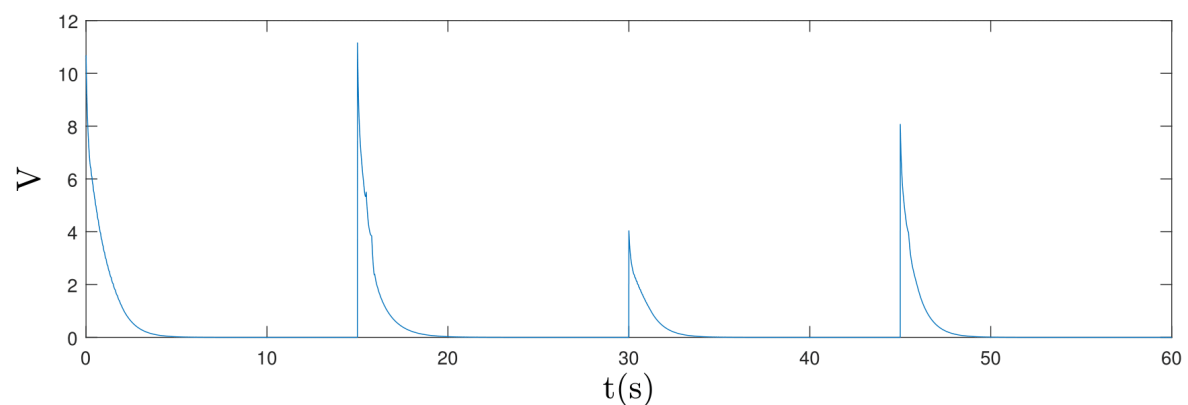

a) Lyapunov function

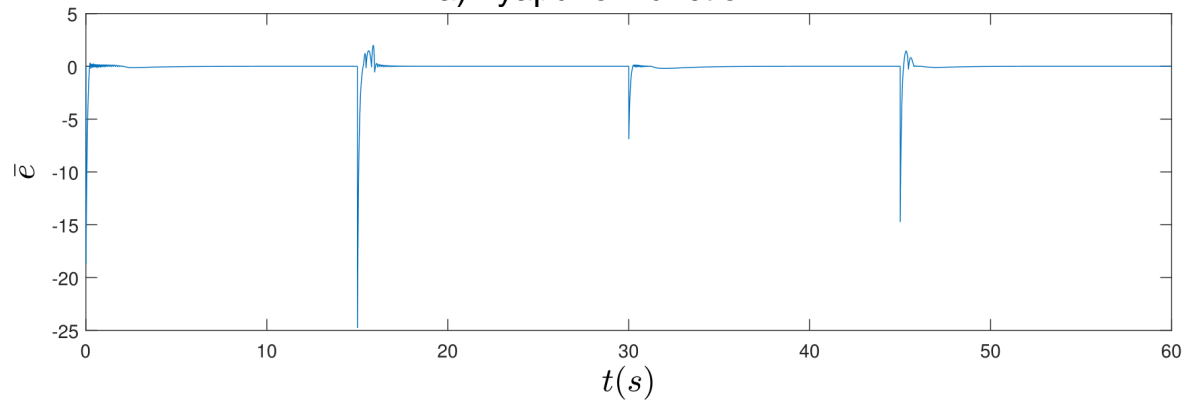

b) Event function

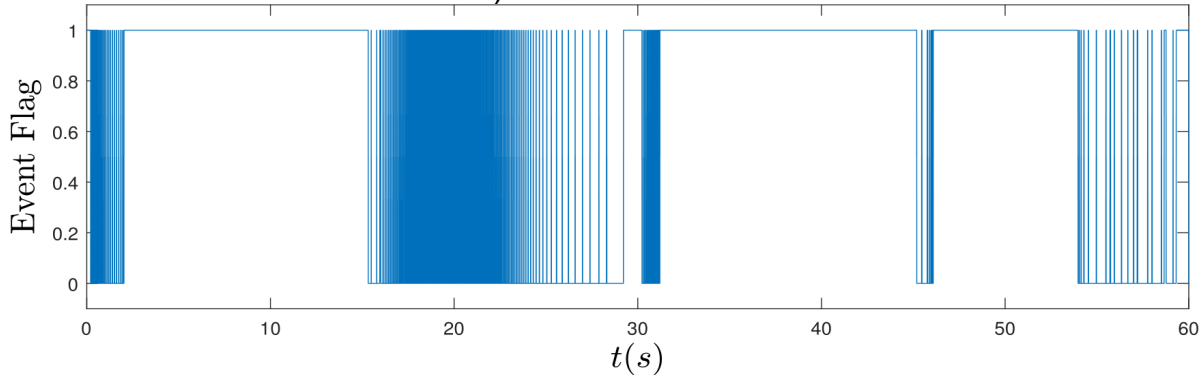

c) Event flag of the event function

Figure 4. Lyapunov function $V$, event-triggered function $\bar{e}$ and event flag of the event function. 


\section{Conclusions}

In the present work a control strategy triggered by events was presented, which was applied in simulation to a robot manipulator of three degrees of freedom. To evaluate the performance of the ETC compared to a CTC, three performance indices were computed. These results showed that the CTC gives a better behavior than the ETC, because the ETC does not require the continuous update of the control signal to perform the regulation task. Therefore, the error in the ETC is larger. On the other hand, based on the tests performed, it was found that the ETC showed an acceptable performance with asymptotic convergence, obtaining some benefits without significantly affecting the performance of the system.

Furthermore, control signal updates required by ETC is reduced by $32.8389 \%$ compared with CTC based on the classical method of TTC. Due to computational calculation is reduced, consequently the energy consumption is lower. On the other hand, due to control signal is updated aperiodically, it is possible to process other task in the time when the control signal is not updated.

\section{Acknowledgements}

Authors acknowledge ssupport from the COFAA of the Instituto Politécnico Nacional, via the project number 2018196. The first author is grateful for the Master's scholarship awarded by CONACyT.

\section{References}

Canudas de Wit, C., Siciliano, B., \& Bastin, G. (1996). Theory of Robot Control. London, England.

Chen, Y., Wang, K., Zhai, L., \& Gao, J. (2017). Non-linear model predictive control schemes with application on a 2 link vertical robot manipulator. Robotics and ComputerIntegrated Manufacturing, 3237-3266.

Durand, S., and Guerrero-Castellanos, J. F., Marchand, N., \& Guerrero-Sánchez, W. F. (2013). Event-Based Control of the Inverted Pendulum: Swing up and Stabilization. International Journal of Control, Automation and Systems, 1-10.

Halalchi, H., Bara, G. L., \& Laroche, E. (2010). \{LPV\} Controller Design for Robot Manipulators Based on Augmented $\{$ LMI $\}$ Conditions with Structural Constraints. 4th IFAC Symposium on System, Structure and Control, 289-295.

Kelly, R., Santibáñez, V., \& Loría, A. (2005). Control of Robot Manipulators in Joint Space. London, England: Springer-Verlag.

Marchand, N., Durand, S., \& Guerrero Castellanos, J. F. (2013). A General Formula for Event-Based Stabilization of Nonlinear Systems. IEEE TRANSACTIONS ON AUTOMATIC CONTROL, 1332-1337.

Monaco, S., \& Normand-Cyrot, D. (2007). Advanced Tools for Nonlinear Sampled-Data Systems' Analysis and Control. European Journal of Control, 221-241.

Tripathy, N. S., Kar, I. N., \& Paul, K. (2014). An Event-triggered Based Robust Control of Robot Manipulator. 13th International Conference on Control, Automation, Robotics and Vision, 425-430.

Tso, S. K., \& Lin, L. N. (1996). Neural-Network-Based Adaptive Controller for Uncertainty Compensation of Robot Manipulators. 13th Triennial World Congress, 5001-5006.

Villarreal-Cervantes, M. G., Guerrero-Castellanos, J. F., Ramírez-Martínez, S., \& SánchezSantana, J. P. (2015). Stabilization of a $(3,0)$ mobile robot by means of an eventtriggered control. ISA Transactions, 605-613.

Wilson, J., Charest, M., \& Dubay, R. (2016). Non-linear model predictive control schemes with application on a 2 link vertical robot manipulator. Robotics and ComputerIntegrated Manufacturing, 23-30. 
Zhao, Y., Sheng, Y., \& Liu, X. (2014). A Novel Finite Time Sliding Mode Control for Robotic Manipulators. 19th World Congress The International Federation of Automatic Control, 7336-7341.

\section{Appendix A. Proof of LCF for ETC}

For the regulation problem a change of variable is made, where the new states are given by $\left[\begin{array}{ll}e & \dot{q}\end{array}\right]$ for the system shown in (1), the representation shown in (A.1).

$$
\frac{d}{d t}\left[\begin{array}{l}
e \\
\dot{q}
\end{array}\right]=\left[\begin{array}{c}
\dot{q} \\
M\left(e+q_{d}\right)^{-1}\left[-C\left(e+q_{d}, \dot{q}\right) \dot{q}-G\left(e+q_{d}\right]\right.
\end{array}\right]+\left[\begin{array}{c}
0 \in \mathbb{R}^{3 \times 3} \\
M\left(e+q_{d}\right)^{-1}
\end{array}\right]
$$

Evaluating the time derivative of $V(e, \dot{q})$ through the path (A.1), results in the following:

$$
\begin{aligned}
& \dot{V}=e^{T} P_{1} \dot{q}+\dot{q}^{T} P_{2} \ddot{q}+e^{T} P_{3} \ddot{q}+\dot{q} P_{3} \ddot{q} \\
& =\mathrm{e}^{\mathrm{T}} \mathrm{P}_{1} \dot{\mathrm{q}}+\dot{\mathrm{q}}^{\mathrm{T}} \mathrm{P}_{2}\left[\mathrm{M}^{-1}(-\mathrm{G}-\mathrm{C} \dot{\mathrm{q}}+\mathrm{M} \overline{\mathrm{v}}+\mathrm{C} \dot{\mathrm{q}}+\mathrm{G})\right] \\
& +\mathrm{e}^{\mathrm{T}} \mathrm{P}_{3}\left[\mathrm{M}^{-1}(-\mathrm{G}-\mathrm{C} \dot{\mathrm{q}}+\mathrm{M} \overline{\mathrm{v}}+\mathrm{C \dot {q }}+\mathrm{G})\right]+\dot{\mathrm{q}}^{\mathrm{T}} \mathrm{P}_{3} \dot{\mathrm{q}} \\
& =\mathrm{e}^{\mathrm{T}} \mathrm{P}_{1} \dot{\mathrm{q}}+\dot{\mathrm{q}}^{\mathrm{T}} \mathrm{P}_{2} \overline{\mathrm{v}}+\mathrm{e}^{\mathrm{T}} \mathrm{P}_{3} \overline{\mathrm{v}}+\dot{\mathrm{q}}^{\mathrm{T}} \mathrm{P}_{3} \dot{\mathrm{q}} \\
& =\mathrm{e}^{\mathrm{T}} \mathrm{P}_{1} \dot{\mathrm{q}}+\dot{\mathrm{q}}^{\mathrm{T}} \mathrm{P}_{3} \dot{\mathrm{q}}-\frac{1}{4} \dot{\mathrm{q}}^{\mathrm{T}} \mathrm{P}_{2} R^{-1} P_{3} e-\frac{1}{4} \dot{\mathrm{q}}^{\mathrm{T}} \mathrm{P}_{2} R^{-1} P_{3} \dot{\mathrm{q}}-\frac{1}{4} \mathrm{e}^{\mathrm{T}} \mathrm{P}_{3} R^{-1} P_{3} e \\
& -\frac{1}{4} \mathrm{e}^{\mathrm{T}} \mathrm{P}_{3} R^{-1} P_{3} \dot{\mathrm{q}} \\
& =\left[\begin{array}{l}
e \\
\dot{q}
\end{array}\right]^{T}\left(\left[\begin{array}{ll}
0 & P_{1} \\
0 & P_{3}
\end{array}\right]-\frac{1}{4}\left[\begin{array}{ll}
P_{1} & P_{3} \\
P_{3} & P_{2}
\end{array}\right]\left[\begin{array}{l}
0 \\
I
\end{array}\right]\left[\begin{array}{ccc}
r_{1} & 0 & 0 \\
0 & r_{2} & 0 \\
0 & 0 & r_{3}
\end{array}\right]^{-1}\left[\begin{array}{l}
0 \\
I
\end{array}\right]\left[\begin{array}{ll}
P_{1} & P_{3} \\
P_{3} & P_{2}
\end{array}\right]\right)\left[\begin{array}{l}
e \\
\dot{q}
\end{array}\right] \\
& =\left[\begin{array}{l}
e \\
\dot{q}
\end{array}\right]^{T}\left(\frac{1}{2}\left[\begin{array}{ll}
0 & I \\
0 & 0
\end{array}\right]^{T}\left[\begin{array}{ll}
P_{1} & P_{3} \\
P_{3} & P_{2}
\end{array}\right]+\frac{1}{2}\left[\begin{array}{ll}
P_{1} & P_{3} \\
P_{3} & P_{2}
\end{array}\right]\left[\begin{array}{ll}
0 & I \\
0 & 0
\end{array}\right]\right. \\
& \left.-\frac{1}{4}\left[\begin{array}{ll}
P_{1} & P_{3} \\
P_{3} & P_{2}
\end{array}\right]\left[\begin{array}{l}
0 \\
I
\end{array}\right]\left[\begin{array}{ccc}
r_{1} & 0 & 0 \\
0 & r_{2} & 0 \\
0 & 0 & r_{3}
\end{array}\right]^{-1}\left[\begin{array}{l}
0 \\
I
\end{array}\right]\left[\begin{array}{ll}
P_{1} & P_{3} \\
P_{3} & P_{2}
\end{array}\right]\right)\left[\begin{array}{l}
e \\
\dot{q}
\end{array}\right] \\
& =\left[\begin{array}{l}
e \\
\dot{q}
\end{array}\right]^{T}\left[A^{T} P+P A-P B R^{-1} B^{T} P\right]\left[\begin{array}{l}
e \\
\dot{q}
\end{array}\right]
\end{aligned}
$$

Note that (A.2) presents the general form of the Riccati equation $A^{T} P+P A-$ $P B R^{-1} B^{T} P+Q=0$, where $P$ is a solution to the equation. Consequently, it is possible to obtain a matrix $Q$ such that:

$$
\dot{V}(x)=-\left[\begin{array}{ll}
e & \dot{q}
\end{array}\right] Q\left[\begin{array}{ll}
e & \dot{q}
\end{array}\right]^{T}<0
$$

